ARTICLE

DOI: $10.1038 / s 41467-018-03592-3$

OPEN

\title{
Asymmetric electric field screening in van der Waals heterostructures
}

Lu Hua Li (10 ${ }^{1}$, Tian Tian (1) ${ }^{2}$, Qiran Cai ${ }^{1}$, Chih-Jen Shih (1) ${ }^{2}$ \& Elton J.G. Santos (iD ${ }^{3}$

A long-standing challenge facing the combination of two-dimensional crystals into heterojunction is the unknown effect of mixing layer of different electronic properties (semiconductors, metals, insulators) on the screening features of the fabricated device platforms including their functionality. Here we use a compelling set of theoretical and experimental techniques to elucidate the intrinsic dielectric screening properties of heterostructures formed by $\mathrm{MoS}_{2}$ and graphene layers. We experimentally observed an asymmetric field screening effect relative to the polarization of the applied gate bias into the surface. Surprisingly, such behavior allows selection of the electronic states that screen external fields, and it can be enhanced with increasing of the number of layers of the semiconducting $\mathrm{MoS}_{2}$ rather than the semi-metal. This work not only provides unique insights on the screening properties of a vast amount of heterojunction fabricated so far, but also uncovers the great potential of controlling a fundamental property for device applications.

\footnotetext{
${ }^{1}$ Institute for Frontier Materials, Deakin University, Geelong Waurn Ponds Campus, Victoria 3216, Australia. ${ }^{2}$ Institute for Chemical and Bioengineering, ETH Zürich, 8093 Zürich, Switzerland. ${ }^{3}$ School of Mathematics and Physics, Queen's University Belfast, Belfast BT7 1NN, United Kingdom. Correspondence and requests for materials should be addressed to E.J.G.S. (email: e.santos@qub.ac.uk)
} 
T he van der Waals heterostructures (vdWHs) composed of two-dimensional (2D) crystals and precisely assembled in a deterministic order constitutes a remarkable paradigm for promising electronic and optoelectronic applications with enhanced features and performance ${ }^{1-6}$. With the properties of the individual layers being characterized since the discovery of graphene and other $2 \mathrm{D}$ materials ${ }^{7}$, the great challenge is how to combine them in order to obtain unusual physical and chemical phenomena not observed on the original sheets ${ }^{1}$. The continuous development of experimental methods that allow atomically thin materials to be fabricated on-demand and to be placed on desired locations with an unprecedented control and accuracy have opened pioneering avenues to fabricate complex device architectures using regular bottom-up approaches ${ }^{2-4}$. The atomic flatness and lack of dangling bonds at the surface of 2D layered materials, such as graphene, boron nitride $(\mathrm{BN})$ and transition metal dichalcogenides (TMDCs), allow them to form noncovalent interactions with a wide range of materials without the condition of lattice matching that normally heterojunctions would have. One of the first realizations of such vdWHs was the fabrication of BN/graphene interfaces and the probing of their electronic properties using gate bias $^{8}$. Such system remarkably showed that graphene could develop superior electrical properties, achieving levels of performance comparable to those observed with freestanding layers. In this assembly, BN layers work as an underlying substrate screening out graphene from any dangling bonds, corrugations and charge inhomogeneities that are inherent to standards $\mathrm{SiO}_{2}$ surfaces to play a role on its electronic properties ${ }^{8,9}$. A direct extension of this system was the complete encapsulation of graphene, TMDCs and the combination of them between $\mathrm{BN}$ layers $5,6,10,11$. This rapidly showed that such device framework displayed improved transport properties with high-mobilities ${ }^{10}$. It is important to remark that in all these systems electrostatic gating schemes have been one of the main driving force to probe the chemical and physical properties of either isolated 2D materials before assembling, or their vdWHs afterwards. Electric gate bias measurements have become a feasible way to control, induce and manipulate electronic properties of almost any vdW crystals since graphene early days ${ }^{12-14}$. This relatively simple setup allows deep insight into the charge density reorganization between different stacking sequences, and on the electric-field screening, which determines most of the exquisite device properties observed in vdWHs. However, no direct measurements of the electrostatic screening features of different combinations of $2 \mathrm{D}$ materials have been reported yet, which ramp up further understanding and developments of vdWHs into any technological platform.

Here we show that the dielectric properties of an archetypal vdWH, involving graphene and $\mathrm{MoS}_{2}$, displays an asymmetrical behavior relative to the polarization of the applied gate bias into the surface allowing an asymmetric screening. Using electric force microscopy (EFM), we observe that graphene/ $\mathrm{MoS}_{2}$ heterostructures exhibit distinct electric response to external fields with respect to the stacking sequence and the direction of electric field. A large depolarization field is recorded at the $\mathrm{MoS}_{2}$ side, which is shown to be dependent majority on the number of $\mathrm{MoS}_{2}$ layers into the heterostructure. A multiscale theoretical framework is developed for elucidating such stacking-dependent asymmetric electric screening phenomenon in graphene/MoS 2 vdWHs. At the atomistic level, we employed quantum mechanical ab initio simulations based on density functional theory (DFT) using a total energy vdW functional to resolve various electronic properties of the vdWHs, including polarization, charge transfer and band structures. Our results indicate that the asymmetric formation of interfacial dipole moments preferably at the $\mathrm{MoS}_{2}$ facet accounts for the asymmetric response. We further show that such asymmetric behavior can be explained using a classical quantum capacitor model, described by a set of self-consistent electrostatic
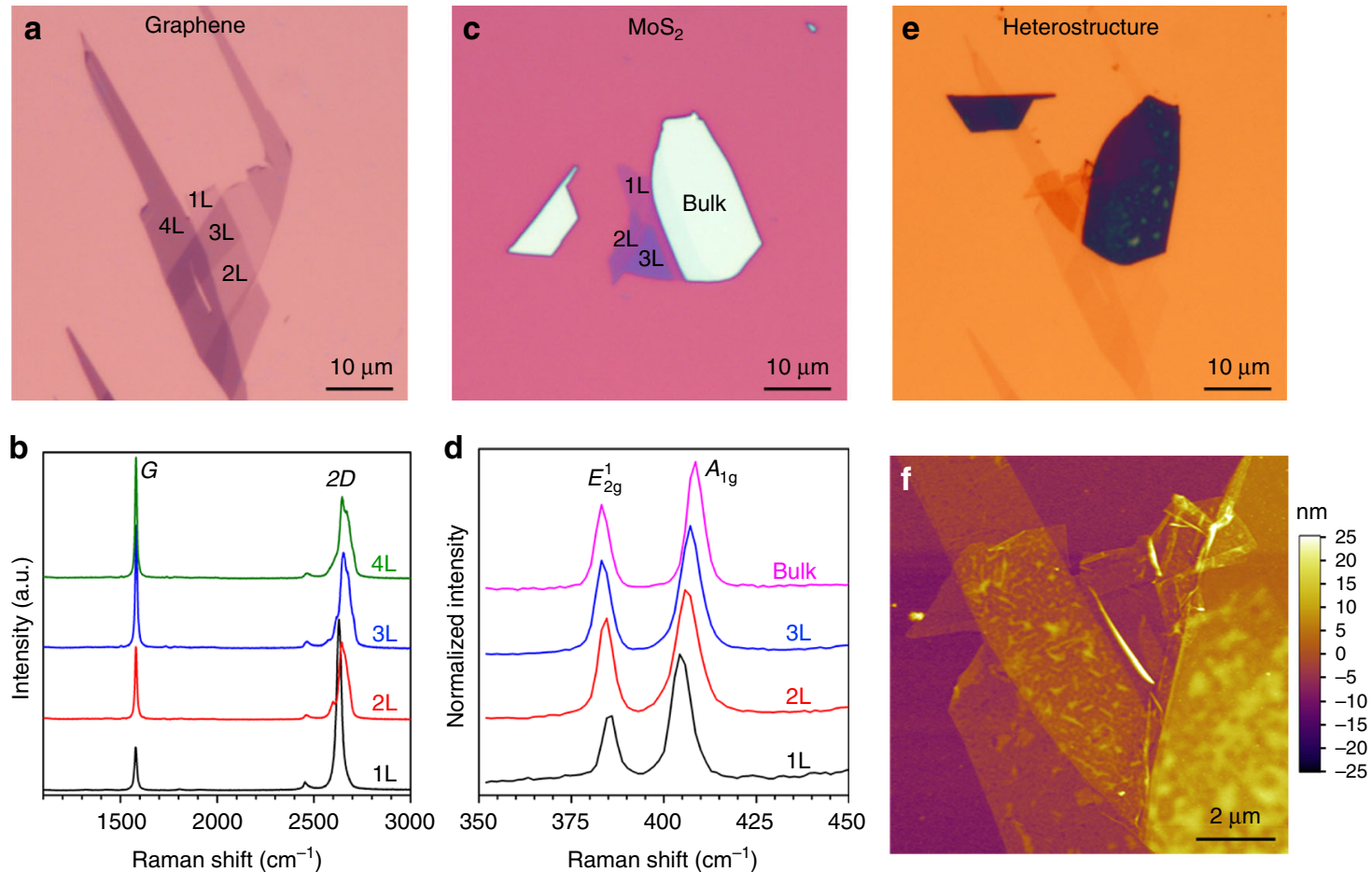

Fig. 1 Sample fabrication and optical characterization. a Optical microscopy photo of the mechanically exfoliated graphene of different thicknesses on 90 $\mathrm{nm} \mathrm{SiO} 2 / \mathrm{Si}$; (b) the corresponding Raman spectra (514.5 nm wavelength); (c) optical microscopy photo of the as-exfoliated $\mathrm{MoS}_{2}$ on $270 \mathrm{~nm} \mathrm{SiO} / 2 \mathrm{Si}$; (d) the corresponding Raman spectra; (e) optical microscopy photo of the $\mathrm{MoS}_{2}$ (top)/graphene (bottom) heterostructures transferred onto $100 \mathrm{~nm}$-thick Au coated $\mathrm{SiO}_{2} / \mathrm{Si}$ by PMMA method; (f) the corresponding AFM image of the heterostructure 
a

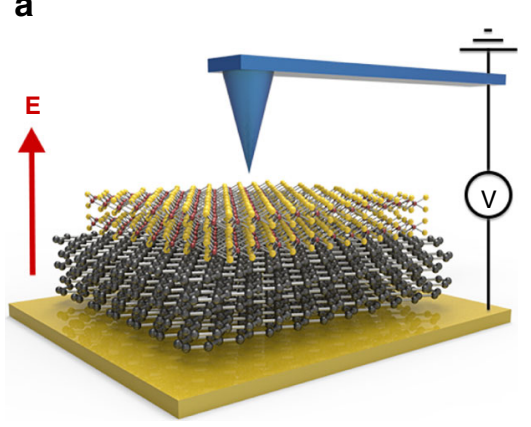

b

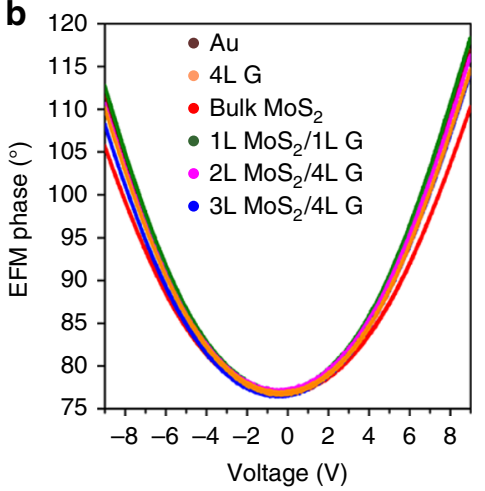

c

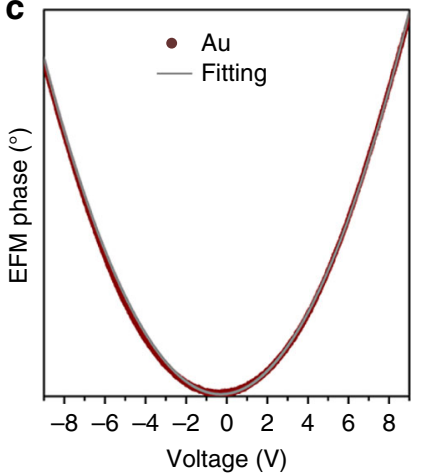

d

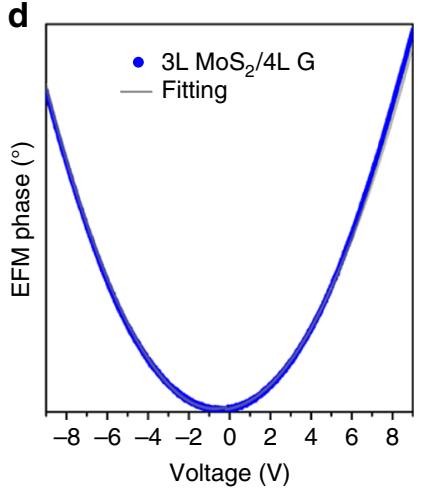

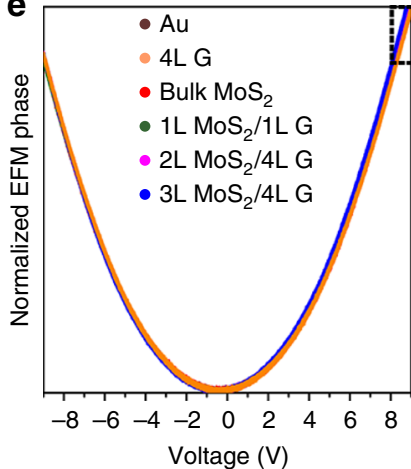

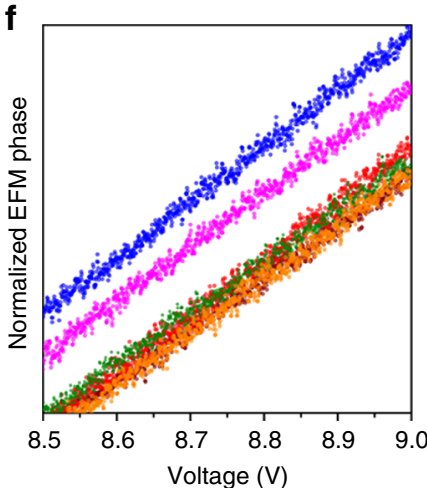

Fig. 2 EFM response of vdW heterostructures under different electric bias. a Drawing of the EFM spectroscopy setup with the Au substrate underneath graphene and $\mathrm{MoS}_{2}$ layers. Positive (negative) bias generates electric fields towards $\mathrm{MoS}_{2}$ (graphene), which are detected by the cantilever. $\mathbf{b}$ The raw EFM data from the six samples under a sweeping substrate DC voltage ( +9 to $-9 \mathrm{~V}$ ); c The excellent fitting of the EFM phase spectrum from the Au substrate (brown) using second-degree polynomials (gray); $\mathbf{d}$ The asymmetry in the EFM phase spectrum from the $3 \mathrm{~L} M o \mathrm{~S}_{2} / 4 \mathrm{~L}$ Graphene heterostructure demonstrated using the same fitting process; e Normalized EFM phase spectra from the six samples to show the asymmetric parabolas obtained from some of the heterostructures; $\mathbf{f}$ Enlarged view of the dashed area in (e)

conservation equations and treating the $2 \mathrm{D}$ layers according to their individual electronic genomes (i.e., energy levels, band gap and quantum capacitance). Our theoretical framework has good consistency with the experimental results at both ab initio and classical levels, showing that the combinations of $2 \mathrm{D}$ materials with distinct electronic structures can result in vdWHs with rich screening features. Furthermore, our theoretical framework is readily applicable for other vdWHs beyond graphene/ $\mathrm{MoS}_{2}$ to explore a wide range of $2 \mathrm{D}$ material combinations with programmable electronic screening properties, which may greatly benefit the design of $2 \mathrm{D}$ vdWH-based functional devices.

\section{Results}

Characterization and measurements. Heterostructures of graphene and $\mathrm{MoS}_{2}$ of different thicknesses on Au coated Si wafer were achieved by two rounds of polymethyl-methacrylate (PMMA) transfer ${ }^{15}$. Figure 1a shows the optical image of $1-4 \mathrm{~L}$ thick graphene nanosheets mechanically exfoliated on a $\mathrm{SiO}_{2}(90$ $\mathrm{nm}$ )/Si substrate. Their thickness was confirmed by the Raman spectra ( $514.5 \mathrm{~nm}$ wavelength) as shown in Fig. 1b. The intensity of the $2 \mathrm{D}$ band of the $1 \mathrm{~L}$ graphene was much stronger than that of the G-band; while the intensity of the $2 \mathrm{D}$ and $\mathrm{G}$ bands of the $2 \mathrm{~L}$ graphene is comparable. The Raman results are consistent with previous reports ${ }^{16}$. The absence of Raman D band suggests the high quality of the graphene. Atomically thin $\mathrm{MoS}_{2}$ nanosheets were exfoliated on $\mathrm{SiO}_{2}(270 \mathrm{~nm}) / \mathrm{Si}$ substrate (Fig. 1c). The portions with different purplish optical contrast gave $E_{2 \mathrm{~g}}^{1}$ and $A_{1 \mathrm{~g}}$ Raman bands centered at 385.3 and $404.7 \mathrm{~cm}^{-1}, 384.3$ and 406.3 $\mathrm{cm}^{-1}$, and 383.6 and $407.2 \mathrm{~cm}^{-1}$, respectively (Fig. 1d). Therefore, they corresponded to $1-3 \mathrm{~L} \mathrm{MoS}_{2}{ }^{17,18}$. To fabricate $\mathrm{MoS}_{2} /$
Graphene heterostructures, the $\mathrm{MoS}_{2}$ was first transferred onto the graphene with the help of PMMA, and then the $\mathrm{MoS}_{2} / \mathrm{Gra}$ phene structure was relocated onto a $100 \mathrm{~nm}$-thick $\mathrm{Au}$ coated $\mathrm{SiO}_{2} / \mathrm{Si}$ substrate, as shown by the optical microscopy photo in Fig. 1e. The corresponding atomic force microscopy (AFM) image of the heterostructure on $\mathrm{Au}$ is displayed in Fig. 1f.

We used EFM to measure the electric field screening properties of the $\mathrm{MoS}_{2} /$ Graphene heterostructures. EFM has been used to investigate the electric field screening in graphene, $\mathrm{BN}$, and $\mathrm{MoS}_{2}$ nanosheets ${ }^{19-21}$. However, the experimental setup in the current study was slightly different from those in these previous reports. We applied a DC voltage sweeping from +9 to $-9 \mathrm{~V}$ to the conductive cantilever, while the Au substrate was grounded, which generated external electric fields of different intensities. The tip of the conductive cantilever was oscillating at its first resonant frequency stayed at a few nanometers above the heterostructures, and acted as a sensor monitoring the capacitance change caused by the change of electric susceptibility of the heterostructures (Fig. 2a). Subtle capacitance changes could be detected by EFM phase shift $(\Delta \phi)$, which can be described as ${ }^{21,22}$.

$$
\Delta \phi=\frac{\partial F / \partial z}{k} \cdot Q_{\text {cant }},
$$

where $\partial F / \partial z$ is the local force gradient, representing the derivative of the electrostatic force felt by the cantilever tip; $k$ is the spring constant of the cantilever; and $Q_{\text {cant }}$ is the $Q$ factor of the cantilever. For simplicity, the interaction between the cantilever tip and the sample in EFM is often viewed as an ideal capacitance (see Supplementary Note 1 and Supplementary Figure 1). Therefore, the local force gradient due to the capacitive 
interaction becomes ${ }^{21,22}$ :

$$
\partial F / \partial z=\frac{1}{2} \frac{\partial^{2} C}{\partial z^{2}}\left(V+V_{\mathrm{CPD}}\right)^{2}
$$

where $C$ and $z$ are the local capacitance and distance between the tip and the sample, respectively; $V$ is the tip voltage; $V_{\mathrm{CPD}}$ is the contact potential difference (CPD) due to the mismatch of the work functions between the tip and the sample. The raw data of the EFM spectroscopy from the Au substrate, 4L graphene, bulk $\mathrm{MoS}_{2}$, and three heterostructures, namely $1 \mathrm{~L} \mathrm{MoS}_{2} / 1 \mathrm{~L}$ Graphene, $2 \mathrm{~L} \mathrm{MoS}_{2} / 4 \mathrm{~L}$ Graphene, and $3 \mathrm{~L} \mathrm{MoS}_{2} / 4 \mathrm{~L}$ Graphene are shown in Fig. 2b. The EFM phase of all the samples formed opening-up parabolas with the axis symmetry parallel with the $y$-axis as a function of the DC voltage. Considering the quadratic function in Eq. 2, we were not surprised that the parabolas were recorded. As $V_{\mathrm{CPD}}$ was fixed for each sample, the formation of the parabolic curves was due to the sweeping $V$. In other words, $V$ was a dominant parameter in our EFM measurements. The opening-up means attractive capacitive interactions under both positive and negative voltages ${ }^{21}$. However, the different samples gave rise to slightly different shapes of the parabolas. This was caused by the other parameters, especially $C$ and $z$. The distance $z$ was inevitably slightly different from sample to sample during the EFM spectroscopy measurements. Although the local capacitance $C$ is very difficult to define, as it depends on many factors, including the shape, size, conductivity, and dielectric property of a sample and cantilever, $C$ was different from sample to sample. Therefore, it is understandable that the different samples resulted in the slightly different parabolic shapes in EFM spectroscopy.

Intriguingly, we found that the parabolas of the EFM phase from some heterostructures lacked the mirror-symmetry as that of a perfect parabola. We give typical examples on symmetric and asymmetric EFM phase in Fig. 2c, d, respectively. The EFM data from the Au substrate could be well fitted by the second-degree polynomial (gray vs. brown in Fig. 2c), but the same fitting process was not able to reproduce the right part (positive bias) of the EFM phase curve from $3 \mathrm{~L} \mathrm{MoS}_{2} / 4 \mathrm{~L}$ Graphene (gray vs. blue in Fig. 2d). To compare this phenomenon from the different samples, we normalized all the EFM data, as shown in Fig. 2e. The enlarged view from the dashed area in Fig. 2e is displayed in Fig. $2 \mathrm{f}$. Similar to the Au substrate, the $4 \mathrm{~L}$ graphene, bulk $\mathrm{MoS}_{2}$, and $1 \mathrm{~L} \mathrm{MoS}_{2} / 1 \mathrm{~L}$ Graphene gave rise to symmetric parabolas of the EFM phase; in contrast, the heterostructures of few-layer $\mathrm{MoS}_{2}$ and graphene, i.e., $2 \mathrm{~L} \mathrm{MoS} / 4 \mathrm{~L}$ Graphene and $3 \mathrm{~L} \mathrm{MoS} / 4 \mathrm{~L}$ Graphene, showed deviated-parabolic EFM phase values under more positive voltages. These EFM results were peculiar. As discussed previously, most of the parameters determining EFM phase, except $V$, should be constant during each measurement. The asymmetric parabolic EFM curves suggest that the electric field screening properties of $2 \mathrm{~L} \mathrm{MoS}_{2} / 4 \mathrm{~L}$ Graphene and $3 \mathrm{~L} \mathrm{MoS}_{2} /$ $4 \mathrm{~L}$ Graphene were probably not constant under different voltages, i.e. external electric fields. In turn, the local capacitance $C$ should change slightly accordingly. This phenomenon was not shown in Au film, 4L graphene, bulk $\mathrm{MoS}_{2}$, or $1 \mathrm{~L} \mathrm{MoS}_{2} / 1 \mathrm{~L}$ Graphene, but became prominent in the heterostructures with increased thickness of graphene and $\mathrm{MoS}_{2}$. We tried to qualitatively analyze the EFM results to estimate the change of electric susceptibility $(\chi)$ of the $3 \mathrm{~L} \mathrm{MoS}_{2} / 4 \mathrm{~L}$ Graphene heterostructure (Supplementary Figure 2). The behavior displays an increment of $\chi$ with the bias pointing to the $\mathrm{MoS}_{2}$ surface. In the following sections, we will show that such asymmetric behavior can be explained at two theoretical levels, both by ab initio simulations with vdW-functionals, as well as a quantum-capacitance-based classical electrostatic model.
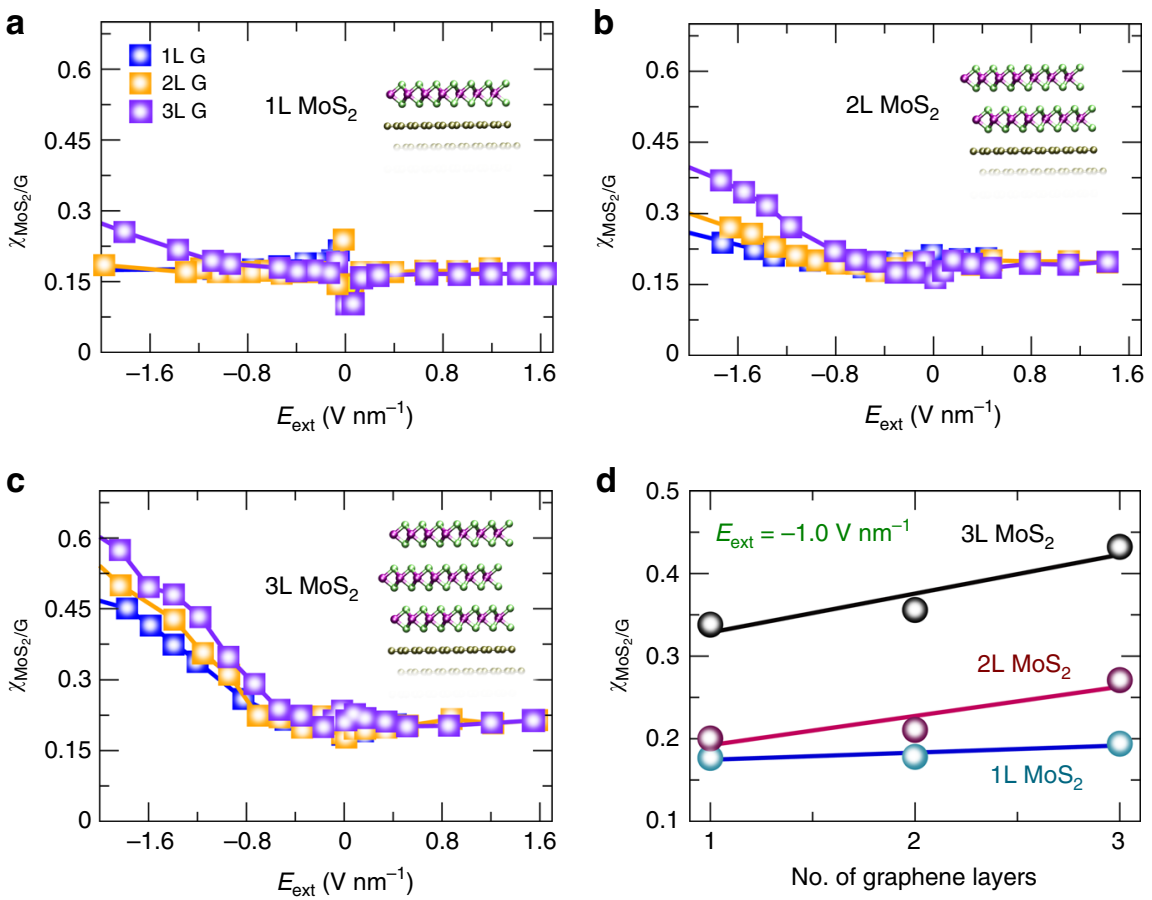

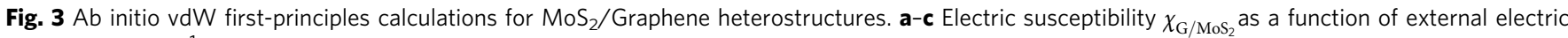
fields $E_{\text {ext }}\left(\mathrm{V} \mathrm{nm}^{-1}\right)$ at $1 \mathrm{~L}, 2 \mathrm{~L}$ and $3 \mathrm{~L} \mathrm{MoS}_{2}$, respectively (see insets). The number of graphene layers systematically increases in each panel at a fixed number of $\mathrm{MoS}_{2}$ sheets following the labeling shown in (a). The polarization of the field follows the orientation in Fig. 2a, where positive (negative) fields go towards graphene $\left(\mathrm{MoS}_{2}\right)$ firstly. $\mathbf{d} \chi_{\mathrm{G} / \mathrm{MoS}_{2}}$ as a function of the number of graphene layers at a static field of $-1.0 \mathrm{~V} n \mathrm{~m}^{-1}$. Different curves correspond to different number of $\operatorname{MoS}_{2}$ layers on the each vdW-heterostructures. Straight lines are fitting curves using a linear equation $\left(\chi=A_{0}+A_{1} N\right)$, where the angular coefficients are: $1 \mathrm{~L}(0.008), 2 \mathrm{~L}(0.035)$ and $3 \mathrm{~L}(0.047)$ 

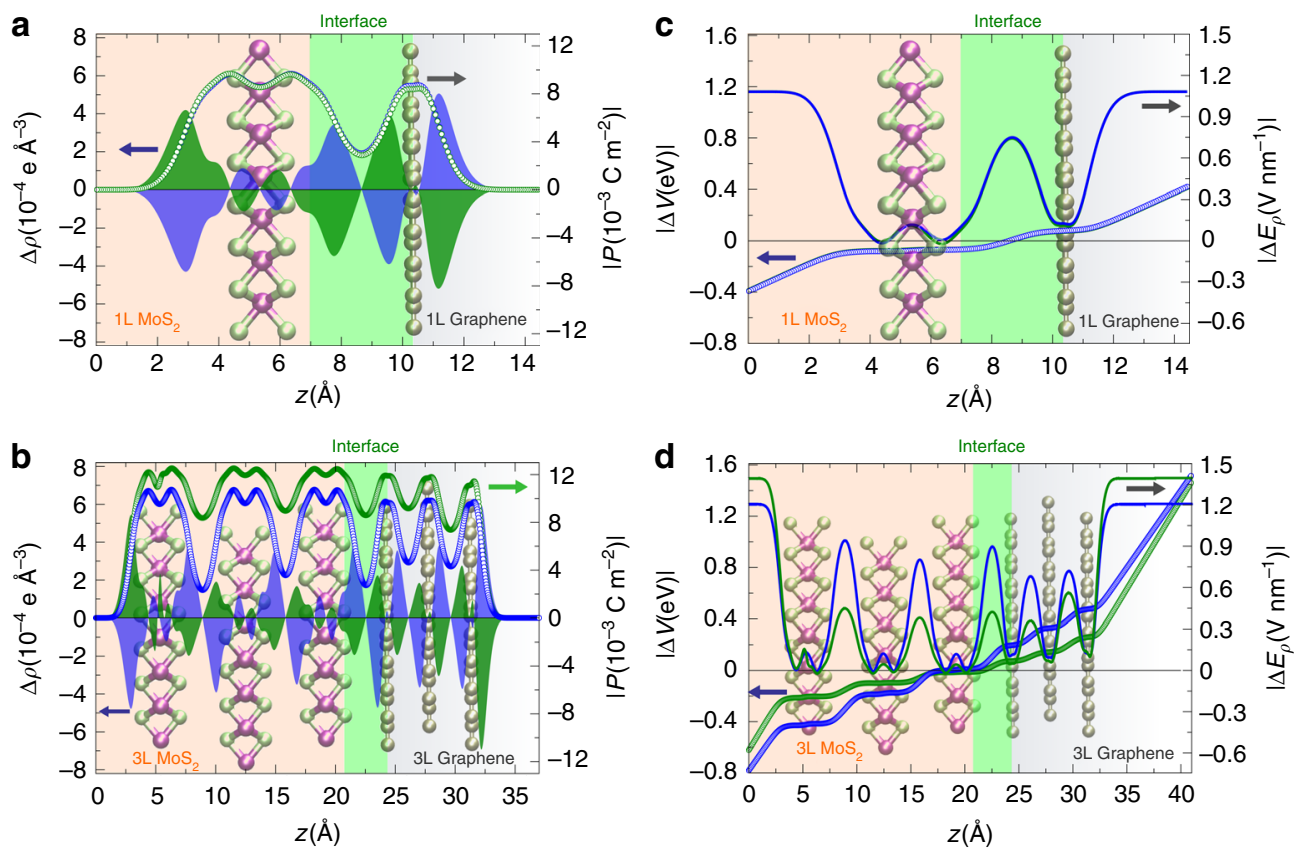

Fig. 4 Asymmetric dipolar contributions at the $\mathrm{MoS}_{2} /$ Graphene interface. a, $\mathbf{b}$ Induced charge density $\Delta \rho\left(10^{-4} \mathrm{e} \AA^{-3}\right)$ (left $y$-axis) and electric polarization $\mid P\left(10^{-3} \mathrm{Cm}^{-2} \mid\right.$ (right $y$-axis) for $1 \mathrm{~L} \mathrm{MoS}_{2} / 1 \mathrm{~L}$ Graphene and $3 \mathrm{~L} \mathrm{MoS} / 3 \mathrm{~L}$ Graphene, respectively. The applied electric field is $\pm 1.0 \mathrm{Vmm}{ }^{-1}$. Blue (green) curves correspond to positive (negative) fields. Positive (negative) fields go towards graphene $\left(\mathrm{MoS}_{2}\right.$ ), and vice versa. The $\mathrm{MoS}_{2} / \mathrm{Graphene}$ interface is highlighted to show the unbalanced formation of electric dipole moments between graphene and $\mathrm{MoS}_{2}$ accordingly with the number of layer layers used to form the heterostructures. (c-d) Difference in electrostatic potential $\Delta V(\mathrm{eV})=V\left(E_{\mathrm{ext}} \neq 0\right)-V\left(E_{\mathrm{ext}}=0\right)$ in the slabs with and without the external electric field of $\pm 1.0 \mathrm{~V} \mathrm{~nm}^{-1}$, and their corresponding response field $\Delta E_{\rho}\left(\mathrm{V} \mathrm{nm}^{-1}\right)$ for $1 \mathrm{~L} \mathrm{MoS}_{2} / 1 \mathrm{~L}$ Graphene and $3 \mathrm{~L} \mathrm{MoS}_{2} / 3 \mathrm{~L} \mathrm{Graphene,} \mathrm{respectively.} \mathrm{The} \mathrm{absolute}$ values of $\left|\Delta E_{\rho}\right|$ and $|\Delta V|$ are taking in (c) and (d) for comparison at the same side of the plot. Geometries for all systems are highlighted at the background of each panel in opacity tone

Quantum mechanical first-principle simulations. To better understand this intriguing phenomenon, we performed two levels of theretical analysis using quantum mechanical ab initio calculations based on density functional theory (DFT); and a classical electrostatic approach using a capacitance model based on charge conservation equation solved variationally (see Methods for details). We first address the quantum mechanical part of the $\mathrm{MoS}_{2} /$ Graphene heterostructures. We calculated the degree of polarization in the $\mathrm{vdW}$ heterostructures of graphene and $\mathrm{MoS}_{2}$ of different number of layers in response to the applied electric fields in terms of the electric susceptibility $\chi_{\mathrm{G} / \mathrm{MoS}_{2}}$. We used the quantum mechanical model presented in refs. ${ }^{23,24}$, where a fully based ab initio approach was employed to extract information about the dielectric response at finite-electric fields and large supercells. No external parameters apart from the magnitude of the external electric fields were utilized in a selfconsistent calculation. The simulations also took into account $\mathrm{vdW}$ dispersion forces, electrostatic interactions, and exchangecorrelation potential within DFT at the same footing.

Figure $3 \mathrm{a}-\mathrm{c}$ show the variation of $\chi_{\mathrm{G} / \mathrm{MoS}_{2}}$ with the electric field at $1 \mathrm{~L}, 2 \mathrm{~L}$ and $3 \mathrm{~L} \mathrm{MoS}_{2}$, respectively, but with a distinct number of graphene layers. Strikingly, only negative bias affected $\chi_{\mathrm{G} / \mathrm{MoS}_{2}}$ despite of the number of graphene and $\mathrm{MoS}_{2}$ sheets present in the heterostructures. This is in remarkable agreement with the experimental results, where an asymmetrical response was recorded only from graphene and few-layer $\mathrm{MoS}_{2}$ heterostructures (Fig. 2f and Supplementary Figure 2). The effect is enhanced, as more $\mathrm{MoS}_{2}$ layers are included into each graphene system. The largest increment is noticed on $3 \mathrm{~L} \mathrm{MoS}_{2} / 3 \mathrm{~L}$ Graphene, where a four-fold enhanced magnitude of $\chi_{\mathrm{G} / \mathrm{MoS}_{2}}$ relative to zero field was calculated (Fig. 3c). We also observed that graphene layers have minor contributions to the effect, as $\chi_{\mathrm{G} / \mathrm{MoS}_{2}}$ slightly varied as more graphene was putted together on top of $\mathrm{MoS}_{2}$ (Fig. 3d). At a fixed value of the electric field ( -1.0 $\mathrm{V} / \mathrm{nm}$ ), larger asymmetric screening was displayed as the number of $\mathrm{MoS}_{2}$ layers increased: the screening in the heterostructures containing $3 \mathrm{~L} \mathrm{MoS}_{2}$ was almost doubled that of $1 \mathrm{~L}$ and $2 \mathrm{~L} \mathrm{MoS}_{2}$ heterostructures. The slope of $\chi_{\mathrm{G} / \mathrm{MoS}_{2}}$ vs. the number of graphene layers also increased with the thickness of $\mathrm{MoS}_{2}$ (1L (0.008), 2L (0.03) and 3L (0.05)), which indicates that thicker $\mathrm{MoS}_{2}$ tends to be more correlated with variations in the number of graphene layers. This follows the behavior observed from EFM measurements, which heterostructures involving thicker $\mathrm{MoS}_{2}$ sheets in contact with graphene gave rise to a more asymmetric EFM phase parabola (Fig. 2f). On the basis of these results, it becomes clear that the transition metal dichalcogenide layers play a key role on this screening effect. We will analyze in the following the modifications of the electronic structure of the heterostructures at finite electric fields, and elucidate the origin of this asymmetric susceptibility dependence on the external bias.

Figure 4 shows that the behavior of $\chi_{\mathrm{G} / \mathrm{MoS}_{2}}$ with the electric bias results from the asymmetrical polarization $P$ associated to which side of the heterostructure the field interacts first. At negative bias, a larger amount of induced charge $\Delta \rho$ was displaced towards the surface-layers of the $\mathrm{MoS}_{2}$ in the heterostructure, which consequently generates a polarization $P$ that provided a better screening to the external electric fields relative to positive bias (Fig. 4a, b). As the number of $\mathrm{MoS}_{2}$ layer is small, little differences are noticed under the reversed electric field, as the dipole moment formed at the interface roughly compensated each other (Fig. 4a). This effect is enlarged, as thicker $\mathrm{MoS}_{2}$ sheets are included. This was due to the amount of interfacial charge redistribution, which generated electric dipole moments preferentially aligned along one direction (Fig. 4b). Electric fields pointing towards graphene were not well screened as those towards $\mathrm{MoS}_{2}$ layers because the induced polarization was not so 
a

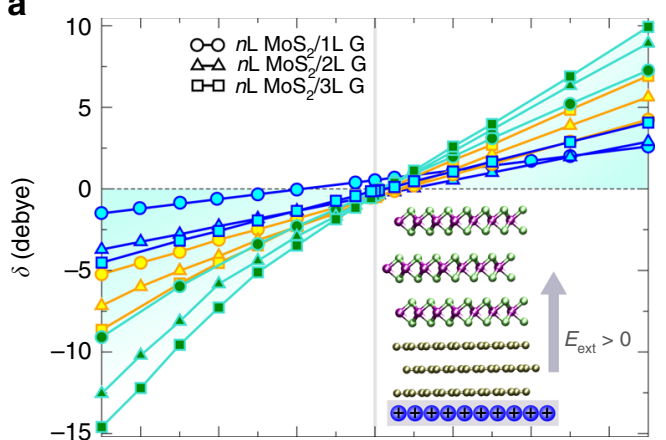

b
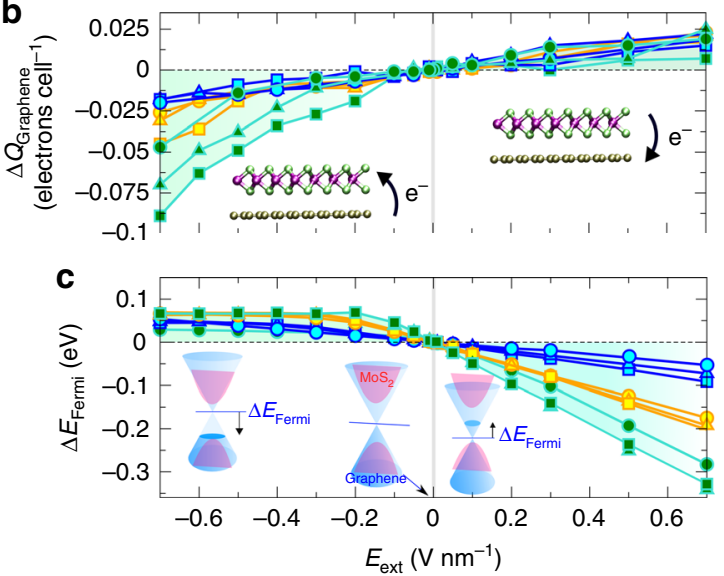
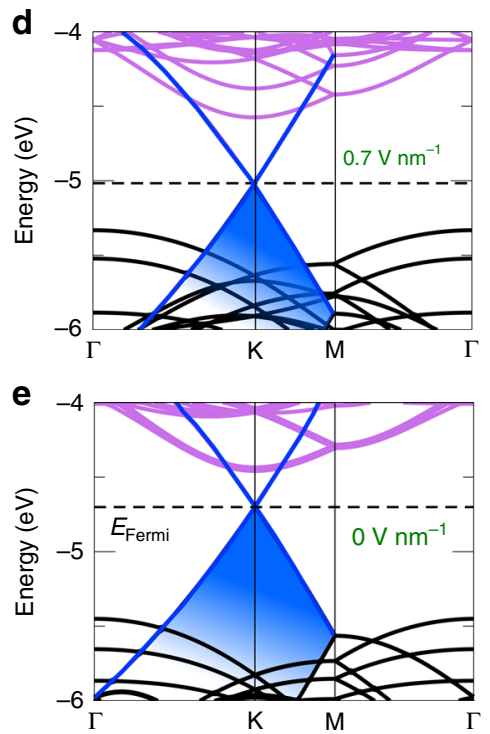

f

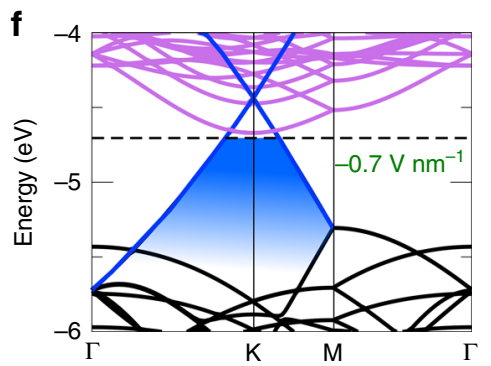

Fig. 5 Asymmetric electronic response under external electric fields. a-c Electric dipole moment $\delta$ (Debye), charge transfer $\Delta Q_{\mathrm{Graphene}}$ (electrons per cell), between graphene and $\mathrm{MoS}_{2}$, and Fermi level variation, $\Delta E_{\mathrm{Fermi}}(\mathrm{eV})$, as a function of electric field $E_{\mathrm{ext}}\left(\mathrm{V}\right.$ nm $\left.{ }^{-1}\right)$, respectively. The different curves and colors correspond to the different number of graphene and $\mathrm{MoS}_{2}$ layers. Blue $(1 \mathrm{~L} \mathrm{MoS})$, orange $(2 \mathrm{~L} \mathrm{MoS})$ and green $\left(3 \mathrm{~L} M o S_{2}\right)$. The same labeling in (a) for the number of graphene layers and colors for the $\mathrm{MoS}_{2}$ are used throughout the different plots. Positive (negative) fields point towards graphene

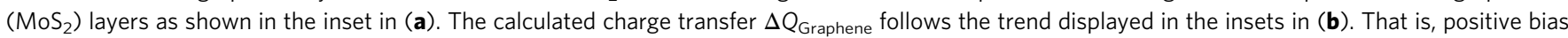
induces charge transfer from $\mathrm{MoS}_{2}$ to graphene, and vice versa. The insets in (c) summarize the effect of the electric field on $\Delta E_{\mathrm{Fermi}}(\mathrm{eV})$ and the resulting electronic structure: positive (negative) bias shifts downward (upward) in energy the Fermi level. Also notice the relative shifts of graphene and $\mathrm{MoS}_{2}$ states with the electric bias. d-f Electronic band structures of the $3 \mathrm{~L} \mathrm{MoS}_{2} / 1 \mathrm{~L}$ Graphene heterostructure at different gate bias. Graphene states are highlighted in blue and $\mathrm{MoS}_{2}$ bands in faint pink. Fermi level is shown by the dashed-line in each panel. An asymmetrical dependence of the electronic properties with the electric field is noted in all calculated quantities

efficient to generate response fields $\Delta E_{\rho}$ that would shield the heterostructures completely. This means that higher magnitudes of electric field were observed inside thinner heterostructures, rather than thicker ones (Fig. $4 \mathrm{c}, \mathrm{d}$ ). We also observed that the induced electric potential $|\Delta V|$ shows a smooth variation over the interface, and it is almost independent of the number of layers composing the junction. $|\Delta V|$ displays high magnitudes over fields towards graphene layers, with a change in polarity at the $\mathrm{MoS}_{2}$ layer near the interface with $|\Delta V|=0$ for thicker vdWHs (see Fig. $4 \mathrm{~d}$ ). This indicates that the interfacial-charge balance in both systems that generates $|\Delta V|$ is sensitive to the amount of polarization charge from the $\mathrm{MoS}_{2}$ layers. That is, the thicker the $\mathrm{MoS}_{2}$ sheets, the larger the polarization. A consequence of this electric field direction-dependent polarization is noted in the different magnitudes of $\Delta E_{\rho}$ observed in the vacuum region outside of $3 \mathrm{~L} \mathrm{MoS}_{2} / 3 \mathrm{~L}$ Graphene system for positive and negative fields (Fig. 4d). In electrostatic boundary conditions, where the normal component of the displacement field $D$ has to be preserved into the system ${ }^{25}$, it gives:

$$
D=E_{\text {vacuum }}=E_{\text {slab }}+4 \pi P_{\text {slab }},
$$

where $E_{\text {slab }}$ corresponds to the field in the sheets and $P_{\text {slab }}$ to the induced polarization. It is worth noting that $P_{\text {slab }}$ differs to $P$ because the latter is calculated directly from the average induced charge using the Poisson equation and the former directly from the boundary conditions and the input field in the simulations (see "Methods" section for details). For negative fields towards $\mathrm{MoS}_{2}$ layers, the second term on the right-hand side in Eq. 3 involving the polarization is appreciably large, which generates a depolarization or response field $\Delta E_{\rho}$ that would overcome the applied external bias. This resulted in smaller electric fields inside the heterojunction (Fig. 4d). A similar effect is observed to fields directed to graphene layers, but higher in magnitudes inside the sheet due to smaller induced polarization. The polarization at the $\mathrm{MoS}_{2} /$ Graphene heterostructure is therefore a contributing factor in the special screening field effect we measured by EFM.

On the basis of the previous analysis, several implications on the electronic structure of the heterojunction can be foreseen. Figure 5 shows an asymmetric electronic response with the external electric field for several quantities. At negative bias, the induced dipole moments associated to the S-Mo-S bonds displace the charge towards the surface of the $\mathrm{MoS}_{2}$ sheet (Fig. 5a), which generated charge transfer from graphene to $\mathrm{MoS}_{2}$ at the interface (Fig. 5b). This charge rearrangement is smaller for positive fields because of the semiconducting nature of the $\mathrm{MoS}_{2}$ layer with less charge-carriers on its surface, and the semi- 
metallic character of graphene. This results in less polarizable field-dependent facet, smaller charge-transfer from $\mathrm{MoS}_{2}$ to graphene, and consequently better screening. The effect of the electric field can also be noted on the tuning of the Fermi level $\Delta E_{\mathrm{Fermi}}$ relative to the charge-neutrality point when no doping concentration was considered on either graphene or $\mathrm{MoS}_{2}$ (Fig. 5c). Thin systems (e.g., $1 \mathrm{~L} \mathrm{MoS}_{2} / 1 \mathrm{~L}$ Graphene) tend to tune their Fermi level almost linearly with the electric field, which is a property intrinsically present at the pristine layers ${ }^{26-28}$. As the number of $\mathrm{MoS}_{2}$ sheets increased, $\Delta E_{\mathrm{Fermi}}$ displayed

a
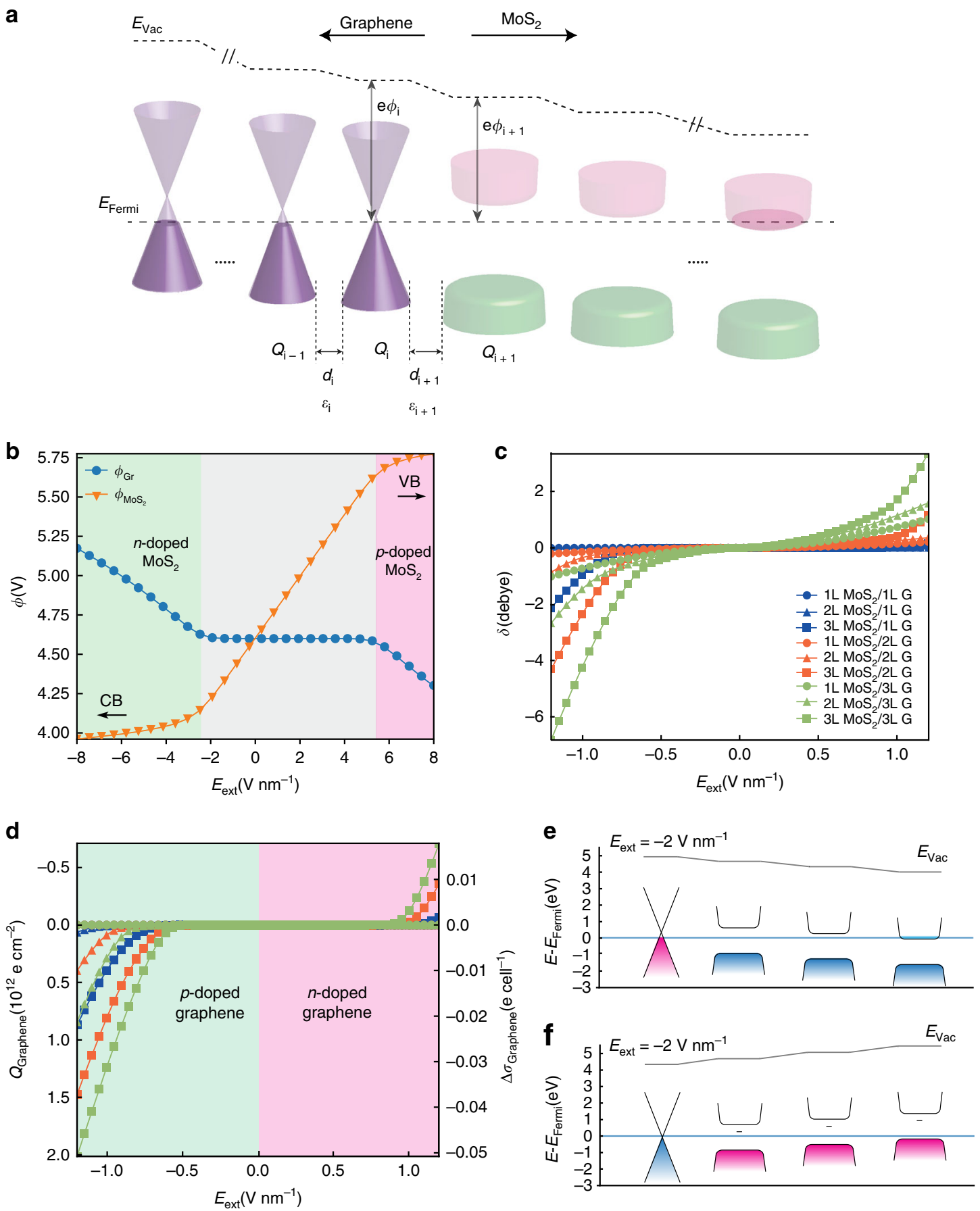

Fig. 6 Classical electrostatic approach for charge redistribution of $\mathrm{MoS}_{2} /$ Graphene vdW heterostructures. a Schematic band diagram of the multilayer $\mathrm{MoS}_{2} /$ Graphene heterostructures. Vacuum level $\left(E_{\text {vac }}\right)$, work function $\left(\phi_{i}\right)$, surface charge density $\left(Q_{i}\right)$, interlayer distance $\left(d_{i}\right)$, and relative permittivity $\left(\varepsilon_{i}\right)$ are shown for each stacking i. b Work functions of graphene and $\mathrm{MoS}_{2}$ as functions of external electric field $E_{\mathrm{ext}}\left(\mathrm{Vnm}^{-1}\right)$. The regimes of the $\mathrm{n}$-doped and p-doped $\mathrm{MoS}_{2}$ are highlighted in green and faint red, respectively. The Fermi level reaches the conduction band (CB) or valence band (VB) of MoS 2 when large negative or positive $E_{\text {ext }}$ is applied, relatively (as shown by the arrows). c Electric dipole moment $\delta$ (Debye) as a function of $E_{\text {ext }}\left(V \mathrm{~nm}{ }^{-1}\right.$ ) for different number of graphene and $\mathrm{MoS}_{2}$ layers on the heterostructures. $\mathbf{d}$ Surface charge density $Q_{\text {Graphene }}$ (left axis) and the amount of charge transfer between graphene and $\mathrm{MoS}_{2}$ layers $\Delta \sigma_{\text {Graphene }}$ (right axis) as a function of $E_{\text {ext }}\left(V_{n m}^{-1}\right)$ for different $\mathrm{MoS}_{2} /$ Graphene systems. The curves follow the labeling in (c). e-f Band alignments for an illustrative case, e.g., $3 \mathrm{~L} \mathrm{MoS}_{2} / 1 \mathrm{~L}$ Graphene under $-2.0 \mathrm{~V} \mathrm{~nm}^{-1}$ and $+2.0 \mathrm{~V} \mathrm{~nm}^{-1}$ electric fields, respectively. Positive charges are shown in faint red and negative charges are shown in blue, respectively. Only band structures near the Fermi level are shown for illustration 
variations at positive fields as large as $0.34 \mathrm{eV}$ for $3 \mathrm{~L} \mathrm{MoS}_{2} / 3 \mathrm{~L}$ Graphene but almost negligible for the negative electric field with the formation of a plateau at $-0.2 \mathrm{~V} \mathrm{~nm}^{-1}$ and beyond. Such asymmetric behavior has been observed when $\mathrm{MoS}_{2}$ layers are used in metal-insulator-semiconductor junctions ${ }^{29}$. Carrier doping induced by the electric field was responsible for the variation of the Fermi level or the work function of $\mathrm{MoS}_{2}$, mainly along one direction, which is directly related to the unbalance of charge density between both sides of the semi-metal and the semiconductor interface. This indicates that the intrinsic character of the electronic structure of each system in vdW heterostructures contributes to the formation of the asymmetric screening observed. This effect has several main implications on the fundamental electronic structure of the $\mathrm{MoS}_{2} / \mathrm{Graphene}$ interfaces as can be appreciated on the band structure calculated at different magnitudes of gate bias for a sample system (e.g. $3 \mathrm{~L}$ $\mathrm{MoS}_{2} / 1 \mathrm{~L}$ Graphene) in Fig. 5d-f. Similar trends are observed for different thicknesses of graphene and $\mathrm{MoS}_{2}$. At $0.0 \mathrm{~V} \mathrm{~nm}^{-1}$, the Fermi level crossed the Dirac point of graphene, as no chargeimbalance was present between both systems. Several $\mathrm{MoS}_{2}$ states at the conduction band were observed at $247.86 \mathrm{meV}$ relative to the Fermi level, which also corresponds to the Schottky barrier presents at the interface ${ }^{30}$. At finite fields, those states are observed to shift up (down) with positive (negative) electric bias, with a consequent split as large as $\sim 0.20 \mathrm{eV}$. This modifies their occupation, as some graphene states can become occupied (positive bias) or unoccupied (negative bias) according to the field polarization. The insets in Fig. $5 \mathrm{c}$ summarize the main effect of the bias on graphene and $\mathrm{MoS}_{2}$ states near the Fermi level. This indicates that for electric fields toward the dichalcogenide layer, the states mainly composed of the conduction band of $\mathrm{MoS}_{2}$ with minor contribution from graphene were responsible for the charge-screening effect and vice versa. This suggests the important role of the interface on the electrical properties of the vdW heterostructures, as the polarity of the electric field can select, which states can screen the system against external bias.

Classical electrostatic approach. Apart from the mighty ab initio approach that gives the full picture of electronic states in the vdWHs, it is more desirable that such asymmetric behavior of the vdWHs can be modeled inexpensively using several key electronic properties from the individual $2 \mathrm{D}$ material layers. Here we show that the asymmetric electronic screening of $\mathrm{MoS}_{2} / \mathrm{Graphene} v \mathrm{dW}$ heterostructures under an external electric field can be well described using a classical electrostatic model, taking the quantum capacitance into consideration ${ }^{31}$. As the $2 \mathrm{D}$ vdWHs are stacked via non-covalent interactions, it is found that the individual properties of $2 \mathrm{D}$ materials can still be largely preserved in their stacked layers, which are coupled by the Coulombic interactions $^{32}$. The idea behind our classical electrostatic model is that each individual layer has its own electronic "genome" (i.e., energy level, band gap, quantum capacitance) extracted from ab initio calculations, which can be used as building blocks in solving the electrostatic conservation equations of the whole vdWH, under the non-coupling assumption. Figure 6a schematically shows the band diagram of the $\mathrm{MoS}_{2} /$ Graphene vdWH used in the classical model. Due to the fact that there is no electron drift in the vdWH at equilibrium (which is consistent with the EFM experimental setup and ab initio configurations), the Fermi level $E_{\text {Fermi }}$ aligns throughout the $\mathrm{MoS}_{2} / \mathrm{Graphene}$ vdWH. For simplicity of the model, we further assume that (i) the density of states (DOS) of individual layer is invariable with the stacking order and the external electric field and (ii) the interlayer distances $\left(d_{i}\right)$ are not affected by the external electric field. Note that although the transition of band structure is ignored in assumption (i), it has been shown that such classical treatment using Coulombic coupling has relatively high consistency with the ab initio simulations ${ }^{32}$. The charge and potential distribution in the $\mathrm{vdWH}$ is solved by several conservation equations in a self-consistent approach $^{33}$ : (i) the charge of individual $2 \mathrm{D}$ layer $Q_{i}$ follows the charge conservation of the vdWH, (ii) $Q_{i}$ is determined by the electric displacement field adjacent to the 2D layer, (iii) $Q_{i}$ determines the work function of layer $i, \phi_{i}$ and (iv) the work function difference between two adjacent layers is determined by the electric displacement field. More details about the mean-field model can be found in Methods. For the simplest case of $1 \mathrm{~L}$ $\mathrm{MoS}_{2} / 1 \mathrm{~L}$ Graphene, we plot in Fig. 6b the work functions $\phi_{i}$ of both materials, as a function of the external electric field strength $\left(E_{\text {ext }}\right)$ ranging from -8 to $+8 \mathrm{~V} \mathrm{~nm}^{-1}$. Highly n-doped and $\mathrm{p}$ doped $\mathrm{MoS}_{2}$ regimes can be found when $E_{\text {ext }}<-2.3 \mathrm{~V} \mathrm{~nm}^{-1}$ or $E_{\text {ext }}>5.5 \mathrm{~V} \mathrm{~nm}^{-1}$, respectively. The fermi level of $\mathrm{MoS}_{2}$ shifts close to its conduction band (CB) or valence band (VB) in both regimes, respectively, which is accounted for the charge accumulation in the vdWH. Note that a noticeable charge accumulation $\left(>10^{12} \mathrm{e} \mathrm{cm}^{-2}\right.$ ) occurs even when the Fermi level of $\mathrm{MoS}_{2}$ is still ca $0.1 \mathrm{eV}$ away from the band edges, due to the fact that the low quantum capacitance of graphene near its intrinsic Fermi level. On the other hand, when $E_{\text {ext }}$ is between -2.3 and $5.5 \mathrm{~V} \mathrm{~nm}$ -1 , the Fermi level of $\mathrm{MoS}_{2}$ lies far away from the band edges, resulting that the vdWH is merely not polarized and the work function of graphene $\phi_{\mathrm{Gr}}$ shows little change with $E_{\mathrm{ext}}$. We find that the polarization of the $\mathrm{MoS}_{2} /$ Graphene $\mathrm{vdWH}$ is more enhanced under negative external electric field, which corresponds with the findings in Fig. 3a. We ascribe such asymmetry to the difference between the electronic structures of graphene and $\mathrm{MoS}_{2}: \mathrm{MoS}_{2}$ is considered as a $n$-type semiconductor with its intrinsic Fermi level $(-4.5 \mathrm{eV})$ closer to its $\mathrm{CB}(-4.0 \mathrm{eV})$ than its VB $(-5.8 \mathrm{eV})^{34-36}$, while graphene has symmetric linear band structure around its Dirac point $(-4.6 \mathrm{eV})^{37}$, all energy levels are compared with the vacuum level that is set at $0 \mathrm{eV}$. Due to their close Fermi level values, little charge transfer occurs between graphene and $\mathrm{MoS}_{2}$ under weak electric field, and the degree of charge transfer is mainly determined by the position of the Fermi level with respect to the CB or VB of $\mathrm{MoS}_{2}$. Following the same procedure, we calculated the dipole moment $\delta$ (Fig. 6c), and the charge density of the graphene layers $Q_{\text {Graphene }}$ (Fig. $6 \mathrm{~d}$ ) for a different number of layers at the $\mathrm{MoS}_{2} /$ Graphene interface. The results from the classical model show good consistency compared with the quantum mechanical $a b$ initio calculations of dipole moment (Fig. 5a) and charge transfer (Fig. 5b): the charge redistribution is more pronounced with increased layer numbers of graphene and $\mathrm{MoS}_{2}$, and the $\mathrm{MoS}_{2}$ layers contributes more to such effect than graphene. Note that for thicker graphene layers (e.g., $\mathrm{nL} \mathrm{MoS}_{2} / 3 \mathrm{~L}$ Graphene), a considerable amount of total dipole moment can still be observed under weak electric field $\left(\left|E_{\text {ext }}\right|<0.5 \mathrm{~V} \mathrm{~nm}^{-1}\right)$, when the charge transfer between graphene and $\mathrm{MoS}_{2}$ is negligible $\left(<10^{11} \mathrm{e} \mathrm{cm}^{-2}\right)$. This indicates that the electric field is well screened by multilayer graphene under such conditions, since the DOS of graphene is finite around the intrinsic Fermi level. The screening in the $\mathrm{MoS}_{2}$ becomes more important only when the Fermi level reach the band edges, that is, when the DOS increases greatly. To verify such statement, we reconstructed the band diagram of $3 \mathrm{~L} \mathrm{MoS}_{2} / 1 \mathrm{~L}$ Graphene system under electric fields of $-2 \mathrm{~V} \mathrm{~nm}^{-1}$ (Fig. 6e) and $+2 \mathrm{~V} \mathrm{~nm}^{-1}$ (Fig. 6f). Under $-2 \mathrm{~V} \mathrm{~nm}^{-1}$ electric field, the Fermi level reaches the $\mathrm{CB}$ out the outermost $\mathrm{MoS}_{2}$ layer, while under $+2 \mathrm{~V} \mathrm{~nm}^{-1}$ electric field, the Fermi level remains within the band gap of $\mathrm{MoS}_{2}$ and has very little shift from the Dirac point of graphene, in good accordance with the ab initio calculations showed in Fig. 5d-f. Charge accumulation occurs mostly on graphene and the outmost $\mathrm{MoS}_{2}$ layer, due to the larger DOS of both layers. 

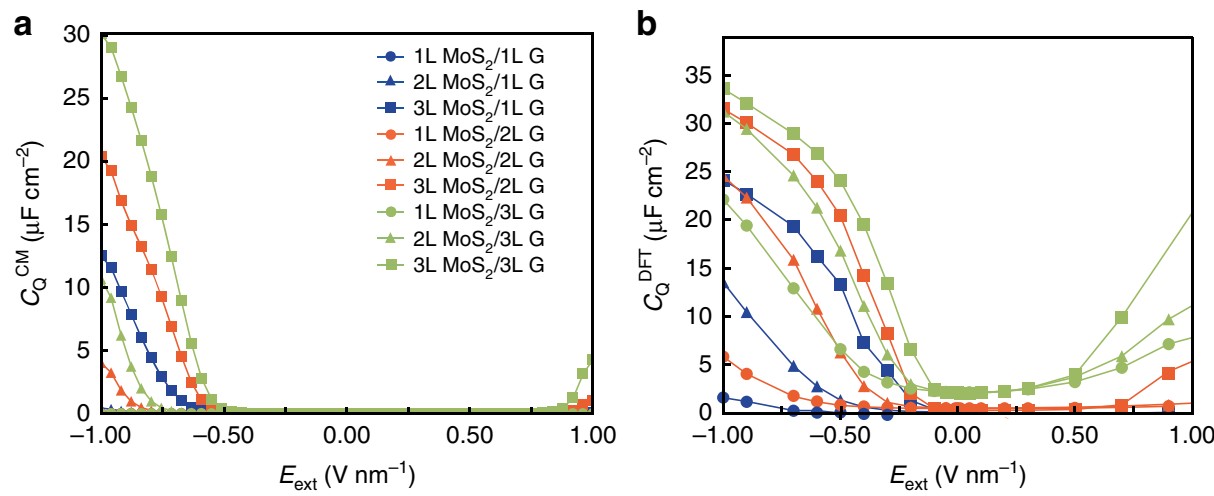

Fig. 7 Quantum capacitance $C_{Q}$ for different $M_{0} S_{2} / G$ raphene heterostructures. a-b Quantum capacitances calculated from the classical model $C_{Q}{ }^{C M}(\mu F$ $\left.\mathrm{cm}^{-2}\right)$ and from the DOS profile of ab initio calculations $C_{Q}{ }^{D F T}\left(\mu F \mathrm{~cm}^{-2}\right)$ as a function of the external field $E_{\text {ext }}\left(V_{n m}{ }^{-1}\right)$ for various MoS $/ G_{r a p h e n e}$ $v d W H s$, respectively. The quantum capacitance increase more under negative electric field than positive field. Both classical and DFT calculations show similar trends of relationship between layer numbers and quantum capacitance: the layer numbers of $\mathrm{MoS}_{2}$ contributes more to the total quantum capacitance than graphene under strong electric field, consistent with the findings of layer-dependent dipole moment and charge transfer

Unifying classical and quantum approaches. The classical electrostatic model shows sound agreement with the ab initio calculations in predicting the asymmetric screening behavior of the $\mathrm{MoS}_{2} /$ Graphene vdWH, as a result of the different energy levels and DOS of both materials. Inspired by the equation of EFM response (Eq. 2), that the local capacitance of the vdWHs can be varied under different electric field (see experimental section), here we further propose that such asymmetry can be described by the quantum capacitance, within both theoretical frameworks. The vdWH can be considered as a capacitor, characterized by the quantum capacitance $\left(C_{\mathrm{Q}}\right)$, which is a function of the DOS at the Fermi level of the whole vdWH: $C_{\mathrm{Q}}=\operatorname{DOS}\left(E_{\mathrm{Fermi}}\right) e^{2}$. The total DOS of the vdWH can be obtained quantum mechanically at high accuracy. On the other hand, the apparent quantum capacitance in the classical model can be calculated by the differentiating the charge density by the potential drop across the vacuum level $\Delta E_{\mathrm{vac}}$ (equivalent to the total difference of work functions $\sum \Delta \phi_{i}$ ): $\mathrm{C}_{\mathrm{Q}}$ $=\partial Q / \partial \Delta E_{\mathrm{Vac}}$. We compare the quantum capacitances calculated by the classical model $\left(\mathrm{C}_{\mathrm{Q}}{ }^{\mathrm{CM}}\right)$ and DFT calculations $\left(\mathrm{C}_{\mathrm{Q}}{ }^{\mathrm{DFT}}\right)$ as functions of $\mathrm{E}_{\text {ext }}$ in various $\mathrm{MoS}_{2} / \mathrm{Graphene}$ systems in Fig. 7a, b, respectively. Interesting, the quantum capacitances calculated by both methods show very similar behavior under external electric field, with the maximum quantum capacitance reaching $\sim 30-33$ $\mu \mathrm{F} \mathrm{cm}^{-2}$ in $3 \mathrm{~L} \mathrm{MoS}_{2} / 3 \mathrm{~L}$ Graphene configuration, under an electric field of $-1 \mathrm{~V} \mathrm{~nm}^{-1}$. We find the layer dependency of quantum capacitance is very similar to that of the dipole moment and charge transfer: the layer number of $\mathrm{MoS}_{2}$ is dominating the magnitude of the total quantum capacitance under strong electric field. This is reasonable due to the higher quantum capacitance of $\mathrm{MoS}_{2}$ than graphene when Fermi level shifts to the band edges ${ }^{33}$. Note that the DFT calculations predict a non-zero quantum capacitance of vdWHs with $2 \mathrm{~L}$ and $3 \mathrm{~L}$ graphene even without external electric field, as a result of the interlayer coupling, which is not included in the classical model. Despite the minute difference between both theoretical frameworks, it is clear that the quantum capacitance of the $\mathrm{MoS}_{2} /$ Graphene $\mathrm{vdWH}$, as a combination of the energy levels and DOS, can describe the asymmetric screening behavior with good precision. Our multiscale theoretical framework is thus readily applicable for a variety of vdWHs beyond graphene/ $\mathrm{MoS}_{2}$, by utilizing the electronic "genome", in particular, the quantum capacitances of individual 2D layers. A full picture of electric screening of $2 \mathrm{D}$ vdWHs can be built benefited from the framework proposed in this work, extending the "tip of the iceberg" of the electrostatic nature of two-layer $2 \mathrm{D}$ vdWHs revealed by recent studies $29,38-40$.

\section{Discussion}

In summary, our findings reveal fundamental knowledge of the screening properties of van der Waals heterostructures using widely used two-dimensional materials, such as graphene and $\mathrm{MoS}_{2}$. Graphene/MoS 2 constitutes an archetypal of vdW heterostructure with exciting possibilities for electronic devices based on atomically thin films. We have shown an asymmetric electric field response on the screening properties of $\mathrm{MoS}_{2} / \mathrm{Graphene}$ stackings via high-resolution EFM spectroscopy and a multiscale theoretical analysis that involve quantum mechanical ab initio density functional theory, including vdW dispersion forces, and a classical electrostatic approach considering the quantum capacitance. Our ab initio calculations are further unified in a quantum capacitance-based model, showing that the difference between the energy levels and band structures between graphene and $\mathrm{MoS}_{2}$ is account for the asymmetric screening behavior. After the transfer of $\mathrm{MoS}_{2}$ on graphene, the screening of either isolated graphene or $\mathrm{MoS}_{2}$ changes accordingly to the sign of the electric bias utilized becoming polarity-dependent. The EFM phase spectrum shows an asymmetry with the tip voltage, as the number of $\mathrm{MoS}_{2}$ layers increases relative to that of the graphene. Electric fields towards $\mathrm{MoS}_{2}$ tend to be better screened than those directed to graphene as an asymmetrical polarization associated with charge transfer at the $\mathrm{MoS}_{2} /$ Graphene interface, generate response fields that opposed to external bias. Such charge rearrangement also polarized the interface inducing the appearance of dipole moments and consequently giving a directional character to the underlying electronic structure. In particular, external fields in such vdW heterostructures can select which electronic states can be used to screen the gate bias, which clearly give an external control on the screening properties according to the stacking order and thickness. Our computational-experimental framework paves the way to understand and engineer the electronic and dielectric properties of a broad class of 2D materials assembled in heterojunctions for different technological applications, such as optoelectronics and plasmonics.

\section{Methods}

Experimental. The graphene and $\mathrm{MoS}_{2}$ nanosheets were mechanically exfoliated by Scotch tape. Highly oriented pyrolytic graphite (HOPG) (Momentive, US) and synthetic $\mathrm{MoS}_{2}$ crystals (2D Semiconductors, USA) were used as received. Si wafers with 90 and $270 \mathrm{~nm}$ thick thermal $\mathrm{SiO}_{2}$ were used for graphene and $\mathrm{MoS}_{2}$, 
respectively. An Olympus optical microscopy (BX51) equipped with a DP71 camera was used to search atomically thin nanosheets, and a Cypher AFM (Asylum Research, US) was employed for topography measurements. The Raman spectra were collected by a Renishaw Raman microscope using $514.5 \mathrm{~nm}$ (for $\mathrm{MoS}_{2}$ ) and $633 \mathrm{~nm}$ (for graphene) lasers and an objective lens of $\times 100$ (a numerical aperture of 0.9). For the fabrication of the heterostructure, the identified $\mathrm{MoS}_{2}$ nanosheets were firstly coated by thin layer of PMMA, then peeled off from the $\mathrm{SiO}_{2} / \mathrm{Si}$ via etching by $\mathrm{NaOH}$, stacked on graphene on $\mathrm{SiO}_{2} / \mathrm{Si}$ under the optical microscope. The $\mathrm{MoS}_{2} /$ Graphene structure was transferred to $\mathrm{Au}(100 \mathrm{~nm})$ coated $\mathrm{SiO}_{2} / \mathrm{Si}$ substrate following a similar procedure. The Au coating was produced by a Leica ACE600 sputter with a crystal balance monitoring the coating thickness in real time. The EFM measurements were conducted on the Cypher AFM. The EFM phase data were collected by a Pt/Ti-coated cantilever with a spring constant of $\sim 2$ $\mathrm{N} / \mathrm{m}$ (ElectricLever, Asylum Research, USA) dwelling above the heterostructure at a sampling rate of $2 \mathrm{kHz}$, while a voltage sweeping linearly from +9 to $-9 \mathrm{~V}$ was applied on the cantilever over a period of $20 \mathrm{~s}$.

Ab initio quantum calculations. Calculations were based on ab initio density functional theory using the SIESTA ${ }^{41}$ and the VASP codes ${ }^{42,43}$. Projected augmented wave method (PAW) ${ }^{44,45}$ for the latter, and norm-conserving (NC) Troullier-Martins pseudopotentials ${ }^{46}$ for the former, have been used in the description of the bonding environment for Mo, S and $\mathrm{C}$. The shape of the numerical atomic orbitals (NAOs) was automatically determined by the algorithms described in ref. ${ }^{41}$. The generalized gradient approximation ${ }^{47}$ along with the $\mathrm{DRSLL}^{48}$ functional was used in both methods, together with a double-zeta polarized basis set in Siesta, and a well-converged plane-wave cutoff of $500 \mathrm{eV}$ in VASP. We have explicitly checked the effect of different modifications on the exchange part of the vdW density functional on the interlayer distance between graphene and $\mathrm{MoS}_{2}$ as shown in Supplementary Table 1. Minor differences were found between DRSLL and other vdW functionals, as the interlayer distance changes by around $2 \%$. This resulted in negligible variations on the charge density (see Supplementary Note 2 and Supplementary Figure 3). The cutoff radii of the different orbitals in SIESTA were obtained using an energy shift of $50 \mathrm{meV}$, which proved to be sufficiently accurate to describe the geometries and the energetics. Atoms were allowed to relax under the conjugate-gradient algorithm until the forces acting on the atoms were less than $1 \times 10^{-8} \mathrm{eV}^{-1}$. The self-consistent field (SCF) convergence was also set to $1.0 \times 10^{-8} \mathrm{eV}$. To model the system studied in the experiments, we created large supercells containing up to 394 atoms to simulate the interface between different number of graphene and $\mathrm{MoS}_{2}$ layers. We have optimized the supercell for the $\mathrm{MoS}_{2} /$ Graphene interface using a $5 \times 5$ graphene cell on a $4 \times 4 \mathrm{MoS}_{2}$ cell, where the mismatch between different lattice constants is smaller than $\sim 2.0 \%$ (i.e. the systems are commensurate). We have kept the lattice constant of the $\mathrm{MoS}_{2}$ at equilibrium, and stretched the one for graphene by that amount. Negligible variations of the graphene electronic properties are observed with the preservation of the Dirac cone for all systems. To avoid any interactions between supercells in the non-periodic direction, a $20 \AA$ vacuum space was used in all calculations. In addition to this, a cutoff energy of 120 Ry was used to resolve the real-space grid used to calculate the Hartree and exchange correlation contribution to the total energy in SIESTA. The Brillouin zone was sampled with a $9 \times 9 \times 1$ grid under the Monkhorst-Pack scheme ${ }^{49}$ to perform relaxations with and without van der Waals interactions. Energetics and electronic band structure were calculated using a converged $44 \times 44 \times 1 \mathrm{k}$-sampling for the unit cell of Graphene/ $\mathrm{MoS}_{2}$. In addition to this we used a Fermi-Dirac distribution with an electronic temperature of $k_{B} T=20 \mathrm{meV}$ to resolve the electronic structure.

The electric field $E_{\text {ext }}=E_{\mathrm{z}}$ across the vdW heterostructures is simulated using a spatially periodic sawtooh-like potential $V(r)=e \mathbf{E} \cdot \mathbf{r}$ perpendicular to the $\mathrm{MoS}_{2} /$ Graphene heterostructures. Such potential is convenient to analyze the response of finite systems (e.g., slabs) to electric fields ${ }^{22,50-55}$, while problematic for extended systems (e.g., bulk). The magnitudes of the spatially varying electrostatic potential $\left\langle V(z)>=\frac{1}{A} \int_{A} V(x, y, z) \mathrm{d} x \mathrm{~d} y\right.$ and charge density $\left\langle\rho(z)>=\frac{1}{A} \int_{A} \rho(x, y, z) \mathrm{d} x \mathrm{~d} y\right.$ across the supercell are determined via a convolution with a filter function to eliminate undesired oscillations and conserve the main features important in the analysis. The variations of both quantities, $\Delta<\rho(z)\rangle=\langle\rho(z)\rangle_{E \neq 0}-\langle\rho(z)\rangle_{E=0}$ and $\Delta\langle V(z)\rangle=\langle V(z)\rangle_{E \neq 0}-\langle V(z)\rangle_{E=0}$ are determined relative to zero field. The polarization $P(r)$ is calculated by the integration of $\Delta<\rho(z)>$ through $\nabla \cdot \mathbf{P}=-\Delta \rho(r) . P_{\text {slab }}$ is defined through $P_{\text {slab }}=\chi E_{\text {slab }}$ where $E_{\text {slab }}=\frac{E_{\text {ext }}}{(1+4 \pi \chi(1-l / c))}$, with $l$ the thickness of the vdW heterostructure, and $c$ the height of the supercell ${ }^{23,24}$

Classical electrostatic model. In the classical model, the graphene and $\mathrm{MoS}_{2}$ layers are treated as individual layers, with the the band structures (band gap, DOS and intrinsic work functions) considered as invariable to external electric field (i.e. the effect interlayer coupling on band structure is neglected). We further consider that the interlayer distance $d_{\mathrm{i}}$ between the i- 1 and i-th layers is fixed, and taken as the interlayer distance in DFT calculations under zero field. The interlayer dielectric constant $\varepsilon_{\mathrm{i}}$ between the $i$-1 and $i$-th layer is considered as uniform. For simplicity, we consider that $\varepsilon_{\mathrm{i}}$ is independent of the external field, while the current model can be easily adapted for field-dependent dielectric constant in multilayer $2 \mathrm{D}$ materials using ab initio calculation results ${ }^{23,24}$. We consider the interlayer electric field $E_{\mathrm{i}}$ to be uniform. The charge density $Q_{\mathrm{i}}$ and work function $\phi_{\mathrm{i}}$ of each layer can thus be solved through the following conservation equations in a selfconsistent way:

The charge neutrality of the vdWH:

$$
\sum_{i} Q_{i}=0
$$

Charge balance of the $i$-th layer by Gauss law:

$$
\varepsilon_{\mathrm{i}} E_{\mathrm{i}}+Q_{\mathrm{i}}-\varepsilon_{\mathrm{i}+1} E_{\mathrm{i}+1}=0
$$

$Q_{\mathrm{i}}$ as a function of the work function $\phi_{\mathrm{i}}$ of $i$-th layer:

$$
\left.Q_{\mathrm{i}}\left(\phi_{\mathrm{i}}\right)=\int_{-\infty}^{\infty} \operatorname{DOS}\left(E^{\prime}\right)\left[f\left(E^{\prime}-e \phi_{\mathrm{i}}\right)\right)-f\left(E^{\prime}-e \phi_{\mathrm{i} 0}\right)\right] \mathrm{d} E^{\prime},
$$

where $f(E)$ is the Fermi-Dirac distribution function, and $\phi_{\mathrm{i} 0}$ is the intrinsic work function of the $i$-th layer.

The potential drop between 2 adjacent layers:

$$
\Delta_{\mathrm{i}}=\phi_{\mathrm{i}+1}-\phi_{\mathrm{i}}=E_{\mathrm{i}} d_{\mathrm{i}} .
$$

Note that for individual layers, $C_{\mathrm{Q}, i}=\operatorname{DOS}_{i} \cdot e^{2}$. We simplify the quantum capacitance of graphene using a linear model: $C_{\mathrm{Graphen}, i}=26.1 \mu \mathrm{F} \mathrm{cm}{ }^{-2} \mathrm{~V}^{-1} \Delta \phi_{i}$, while the quantum capacitance of $\mathrm{MoS}_{2}$ is a step function, where no density of states exist within the band gap, while $C_{\mathrm{Mos}_{2}, \mathrm{n}}=48 \mu \mathrm{F} \mathrm{cm}{ }^{-2}$ and $C_{\mathrm{Mos}_{2}, \mathrm{p}}=$ $180 \mu \mathrm{F} \mathrm{cm}^{-2}$ for $\mathrm{VB}$ and $\mathrm{CB}$, respectively. The intrinsic work function of graphene and $\mathrm{MoS}_{2}$ are set at 4.6 and $4.5 \mathrm{~V}$, respectively. The energy levels of $\mathrm{CB}$ and VB of $\mathrm{MoS}_{2}$ are taken as -4.0 and $-5.8 \mathrm{eV}$, respectively.

Data availability. The data that support the findings of this study are available from the corresponding author upon reasonable request.

Received: 18 December 2017 Accepted: 27 February 2018 Published online: 28 March 2018

\section{References}

1. Geim, A. K. \& Grigorieva, I. V. Van der Waals heterostructures. Nature 499, 419-425 (2013).

2. Jariwala, D., Marks, T. J. \& Hersam, M. C. Mixed-dimensional van der Waals heterostructures. Nat. Mater. 16, 170-181 (2017).

3. Novoselov, K. S., Mishchenko, A., Carvalho, A. \& Neto, A. H. C. 2D materials and van der Waals heterostructures. Science 353, 9439-9458 (2016).

4. Liu, Y. et al. Van der Waals heterostructures and devices. Nat. Rev. Mater. 1, 16042-16059 (2016).

5. Withers, F. et al. Light-emitting diodes by band-structure engineering in van der Waals heterostructures. Nat. Mater. 14, 301-306 (2015).

6. Britnell, L. et al. Field-effect tunneling transistor based on vertical graphene heterostructures. Science 335, 947-950 (2012).

7. Novoselov, K. S. et al. Two-dimensional atomic crystals. Proc. Natl Acad. Sci. USA 102, 10451-10453 (2005).

8. Dean, C. R. et al. Boron nitride substrates for high-quality graphene electronics. Nat. Nanotechnol. 5, 722-726 (2010).

9. Xue, J. et al. Scanning tunnelling microscopy and spectroscopy of ultra-flat graphene on hexagonal boron nitride. Nat. Mater. 10, 282-285 (2011).

10. Cui, X. et al. Multi-terminal transport measurements of $\mathrm{MoS}_{2}$ using a van der Waals heterostructure device platform. Nat. Nanotechnol. 10, 534-540 (2015).

11. Wang, L. et al. One-dimensional electrical contact to a two-dimensional material. Science 342, 614-617 (2013).

12. Novoselov, K. S. et al. Electric field effect in atomically thin carbon films. Science 306, 666-669 (2004)

13. Zhang, Y., Tan, Y.-W., Stormer, H. L. \& Kim, P. Experimental observation of the quantum Hall effect and Berry's phase in graphene. Nature 438, 201-204 (2005).

14. Novoselov, K. S. et al. Two-dimensional gas of massless Dirac fermions in graphene. Nature 438, 197-200 (2005).

15. Minemawari, H. et al. Inkjet printing of single-crystal films. Nature $\mathbf{4 7 5}$, 364-367 (2011).

16. Ferrari, A. C. et al. Raman spectrum of graphene and graphene layers. Phys. Rev. Lett. 97, 187401 (2006)

17. Lee, C. et al. Anomalous lattice vibrations of single- and few-layer $\mathrm{MoS}_{2}$. ACS Nano 4, 2695-2700 (2010).

18. Chakraborty, B., Matte, H. S. S. R., Sood, A. K. \& Rao, C. N. R. Layerdependent resonant Raman scattering of a few layer $\mathrm{MoS}_{2}$. J. Raman Spectrosc. 44, 92-96 (2013). 
19. Datta, S. S., Strachan, D. R., Mele, E. J. \& Johnson, A. T. C. Surface potentials and layer charge distributions in few-layer graphene films. Nano Lett. 9, 7-11 (2009).

20. Castellanos-Gomez, A. et al. Electric-field screening in atomically thin layers of $\mathrm{MoS}_{2}$ : the role of interlayer coupling. Adv. Mater. 25, 899-903 (2013).

21. Li, L. H. et al. Dielectric screening in atomically thin boron nitride nanosheets. Nano Lett. 15, 218-223 (2015).

22. Li, L. H. \& Chen, Y. Electric contributions to magnetic force microscopy response from graphene and $\mathrm{MoS}_{2}$ nanosheets. J. Appl. Phys. 116, 213904-213908 (2014).

23. Santos, E. J. G. \& Kaxiras, E. Electric-field dependence of the effective dielectric constant in graphene. Nano Lett. 13, 898-902 (2013).

24. Santos, E. J. G. \& Kaxiras, E. Electrically driven tuning of the dielectric constant in $\mathrm{MoS}_{2}$ layers. ACS Nano 7, 10741-10746 (2013).

25. Meyer, B. \& Vanderbilt, D. Ab initio study of $\mathrm{BaTiO}_{3}$ and $\mathrm{PbTiO}_{3}$ surfaces in external electric fields. Phys. Rev. B 63, 205426-205434 (2001).

26. Yu, Y.-J. et al. Tuning the graphene work function by electric field effect. Nano Lett. 9, 3430-3434 (2009).

27. Wang, R. et al. Control of carrier type and density in exfoliated graphene by interface engineering. ACS Nano 5, 408-412 (2011).

28. Li, Y., Xu, C.-Y., Zhang, B.-Y. \& Zhen, L. Work function modulation of bilayer $\mathrm{MoS}_{2}$ nanoflake by backgate electric field effect. Appl. Phys. Lett. 103, 33122-33126 (2013).

29. Chu, D., Lee, Y. H. \& Kim, E. K. Selective control of electron and hole tunneling in 2D assembly. Sci. Adv. 3, e1602726 (2017).

30. Yu, L. et al. Graphene/MoS ${ }_{2}$ hybrid technology for large-scale twodimensional electronics. Nano Lett. 14, 3055-3063 (2014).

31. Luryi, S. Quantum capacitance devices. Appl. Phys. Lett. 52, 501-503 (1988).

32. Andersen, K., Latini, S. \& Thygesen, K. S. Dielectric genome of van der Waals heterostructures. Nano Lett. 15, 4616-4621 (2015).

33. Tian, T., Rice, P., Santos, E. J. G. \& Shih, C.-J. Multiscale analysis for fieldeffect penetration through two-dimensional materials. Nano Lett. 16, 5044-5052 (2016).

34. Ochedowski, O. et al. Effect of contaminations and surface preparation on the work function of single layer $\mathrm{MoS}_{2}$. Beilstein J. Nanotechnol. 5, 291-297 (2014).

35. Das, S., Chen, H.-Y., Penumatcha, A. V. \& Appenzeller, J. High performance multilayer mos 2 transistors with scandium contacts. Nano Lett. 13, 100-105 (2013).

36. Lu, C.-P., Li, G., Mao, J., Wang, L.-M. \& Andrei, E. Y. Bandgap, mid-gap states, and gating effects in $\mathrm{MoS}_{2}$. Nano Lett. 14, 4628-4633 (2014).

37. Das Sarma, S., Adam, S., Hwang, E. H. \& Rossi, E. Electronic transport in twodimensional graphene. Rev. Mod. Phys. 83, 407-470 (2011).

38. Lee, C.-H. et al. Atomically thin $\mathrm{p}-\mathrm{n}$ junctions with van der Waals heterointerfaces. Nat. Nanotechnol. 9, 676-681 (2014).

39. Furchi, M. M., Pospischil, A., Libisch, F., Burgdörfer, J. \& Mueller, T. Photovoltaic effect in an electrically tunable van der Waals heterojunction. Nano. Lett. 14, 4785-4791 (2014).

40. Roy, T. et al. Dual-Gated $\mathrm{MoS}_{2} / \mathrm{WSe}_{2}$ van der Waals tunnel diodes and transistors. ACS Nano 9, 2071-2079 (2015).

41. Soler, J. M. et al. The SIESTA method for ab initio order- N materials simulation. J. Phys. Condens. Matter 14, 2745-2767 (2002).

42. Kresse, G. \& Hafner, J. Ab initio molecular dynamics for open-shell transition metals. Phys. Rev. B 48, 13115-13118 (1993).

43. Kresse, G. \& Furthmüller, J. Efficient iterative schemes for ab initio totalenergy calculations using a plane-wave basis set. Phys. Rev. B 54, 11169-11186 (1996).

44. Blöchl, P. E. Projector augmented-wave method. Phys. Rev. B 50, 17953-17979 (1994).

45. Kresse, G. \& Joubert, D. From ultrasoft pseudopotentials to the projector augmented-wave method. Phys. Rev. B 59, 1758-1775 (1999).

46. Troullier, N. \& Martins, J. L. Efficient pseudopotentials for plane-wave calculations. Phys. Rev. B 43, 1993-2006 (1991).

47. Perdew, J. P., Burke, K. \& Ernzerhof, M. Generalized gradient approximation made simple. Phys. Rev. Lett. 77, 3865-3868 (1996).

48. Dion, M., Rydberg, H., Schröder, E., Langreth, D. C. \& Lundqvist, B. I. Van der Waals density functional for general geometries. Phys. Rev. Lett. 92, 246401-246405 (2004).
49. Monkhorst, H. J. \& Pack, J. D. Special points for Brillouin-zone integrations. Phys. Rev. B 13, 5188-5192 (1976).

50. Santos, E. J. G. Carrier-mediated magnetoelectric coupling in functionalized graphene. ACS Nano 7, 9927-9932 (2013).

51. Santos, E. J. G. Electrical spin switch in hydrogenated multilayer graphene. J. Phys. Chem. C. 117, 6420-6425 (2013).

52. Santos, E. J. G. Magnetoelectric effect in functionalized few-layer graphene. Phys. Rev. B 87, 155440-155445 (2013).

53. Tian, T. et al. Doping-driven wettability of two-dimensional materials: a multiscale theory. Langmuir 33, 12827-12837 (2017).

54. Ojeda-Aristizabal, C. et al. Molecular arrangement and charge transfer in $\mathrm{C}_{60} /$ graphene heterostructures. ACS Nano 11, 4686-4693 (2017).

55. Santos, E. J. G. et al. Rotational superstructure in van der Waals heterostructure of self-assembled $\mathrm{C}_{60}$ monolayer on the $\mathrm{WSe}_{2}$ surface. Nansocale 9, 13245-13256 (2017).

\section{Acknowledgements}

We acknowledge valuable discussions with Fritz B. Prinz. C.J.S. and T.T. are grateful for financial support from ETH startup funding. L.H.L. thanks the financial support from Australian Research Council (ARC) via Discovery Early Career Researcher Award (DE160100796). E.J.G.S. acknowledges the use of computational resources from the UK national high performance computing service (ARCHER) for which access was obtained via the UKCP consortium (EPSRC grant ref EP/P022626/1); the UK Materials and Molecular Modeling Hub for access to THOMAS supercluster, which is partially funded by EPSRC (EP/P020194/1). The Queen's Fellow Award through the grant number M8407MPH, the Enabling Fund (A5047TSL), and the Department for the Economy (USI 097) are also acknowledged.

\section{Author contributions}

E.J.G.S. and L.H.L. designed the research. L.H.L. fabricated the samples, perform characterization, and EFM measurements. Q.C. performed Raman characterization. T.T. and C.J.S. developed the capacitor model. E.J.G.S. developed the first-principles calculations and performed analysis. L.H.L., T.T. and E.J.G.S. co-wrote the manuscript with inputs from all authors. All authors contributed to this work, read the manuscript, discussed the results, and all agree to the contents of the manuscript.

\section{Additional information}

Supplementary Information accompanies this paper at https://doi.org/10.1038/s41467018-03592-3.

Competing interests: The authors declare no competing interests.

Reprints and permission information is available online at http://npg.nature.com/ reprintsandpermissions/

Publisher's note: Springer Nature remains neutral with regard to jurisdictional claims in published maps and institutional affiliations.

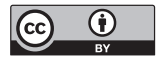

Open Access This article is licensed under a Creative Commons Attribution 4.0 International License, which permits use, sharing, adaptation, distribution and reproduction in any medium or format, as long as you give appropriate credit to the original author(s) and the source, provide a link to the Creative Commons license, and indicate if changes were made. The images or other third party material in this article are included in the article's Creative Commons license, unless indicated otherwise in a credit line to the material. If material is not included in the article's Creative Commons license and your intended use is not permitted by statutory regulation or exceeds the permitted use, you will need to obtain permission directly from the copyright holder. To view a copy of this license, visit http://creativecommons.org/ licenses/by/4.0/.

(C) The Author(s) 2018 